\title{
Vagal stimulation for heart diseases: from animals to men. An example of translational cardiology
}

\author{
P. J. Schwartz
}

Published online: 19 December 2012

(C) Springer Media / Bohn Stafleu van Loghum 2012

\begin{abstract}
A significant series of experimental and clinical studies have demonstrated the close association between reduced vagal reflexes (baroreflex sensitivity, BRS) and increased sudden and non-sudden cardiovascular mortality. Subsequently, evidence was provided that, also among chronic heart failure (HF) patients, depressed BRS is associated with a poorer outcome. At the same time, the encouraging results with experimental and clinical attempts to increase cardiac vagal activity led to a few experimental studies with vagal stimulation (VS) in different models for HF. We first performed a pilot study for VS in HF patients, and then in 2011 we reported the results of a small size multicentre clinical trial. The 6-month and 1-year results are encouraging for feasibility, safety and appear to have a favourable clinical effect. An ongoing large clinical trial will provide a definitive assessment of the efficacy and usefulness of chronic VS in HF patients.
\end{abstract}

Keywords Autonomic nervous system $\cdot$ Baroreflex sensitivity $\cdot$ Heart failure $\cdot$ Vagal stimulation

\author{
P. J. Schwartz \\ Department of Molecular Medicine, University of Pavia, Pavia, Italy \\ P. J. Schwartz $(\bowtie)$ \\ Department of Cardiology, \\ Fondazione IRCCS Policlinico S. Matteo, V. le Golgi, 19, \\ 27100 Pavia, Italy \\ e-mail: peter.schwartz@unipv.it \\ P. J. Schwartz \\ Cardiovascular Genetics Laboratory, Hatter Institute \\ for Cardiovascular Research in Africa, Department of Medicine, \\ University of Cape Town, Cape Town, South Africa \\ P. J. Schwartz \\ Department of Family and Community Medicine, \\ College of Medicine, King Saud University, Riyadh, Saudi Arabia
}

The objective of this essay is to review some aspects of a novel approach to the management of chronic heart failure, a topic recently discussed several times [1-3]. Accordingly, it seems reasonable to tackle this issue by reviewing how this concept was developed and how, from a series of experimental studies with a different objective, the end result was the use of chronic vagal stimulation in patients with heart failure.

In the early 1980 s my interest was largely directed toward the possibility of reducing the risk of developing ventricular fibrillation associated either to arrhythmogenic diseases of genetic origin, such as the long-QT syndrome, or acute myocardial ischaemia. My background, single fibre recording from the sympathetic nerves [4] and from the right cardiac branch of the vagus [5], helped me to develop - with excellent partners such as George Billman and the late Lowell Stone - an animal model for sudden cardiac death that over the years has provided a large number of findings of not minor clinical relevance [6]. We studied conscious dogs with a healed anterior wall myocardial infarction; 1 month after the infarct they performed an exercise stress test and during the last minute we produced a transient episode of acute myocardial ischaemia in conditions of physiologically elevated sympathetic activity, by inflating a balloon occluder previously positioned around the circumflex coronary for $2 \mathrm{~min}$. Under these conditions approximately $50 \%$ of the animals developed ventricular fibrillation (and were defined as 'susceptible' to sudden death) and the remainder did not ('resistant' to sudden death). As the animals were running with defibrillator paddles ligated to the chest, a prompt countershock could re-establish sinus rhythm within seconds and the animals could be studied again over long periods of time. A critical feature of this model is the extremely high reproducibility of the outcome: the susceptible dogs continued to develop ventricular fibrillation whenever exposed to the same 'exercise and ischaemia' challenge and the resistant dogs continued to survive without arrhythmias. 
A conceptual turning point was the realisation that the two groups, susceptible and resistant, could be identified prior to the exercise and ischaemia test. Indeed, the susceptible dogs had markedly reduced baroreflex sensitivity following the phenylephrine test, a marker of reduced ability to reflexly increase vagal activity. We confirmed this experimental finding in a large prospective clinical study conducted in 1284 post-MI patients in which we demonstrated that impaired ability to increase vagal activity was predicting cardiac mortality in patients with ischaemic heart disease [7]. These studies provided the evidence that when vagal activity is reduced, the risk for life-threatening arrhythmias, sudden death, and cardiac mortality increases. They also provided the rationale for exploring the possibility that vagal stimulation might reduce the propensity for VF during acute myocardial ischaemia in clinically relevant conditions. Such a possibility had already been demonstrated in anesthetised preparations $[8,9]$. We developed chronically implantable electrodes to stimulate the right vagus and then designed a case-control study using our sudden death animal model [10]. From a group of 161 dogs, we identified 59 that survived the exercise ischaemia test after having developed VF [10]. They were randomised to repeat the test either in control condition or with vagal stimulation beginning $15 \mathrm{~s}$ after onset of coronary occlusion, while the dogs were running. Whereas $92 \%$ of the control animals had a recurrence of VF, this happened in only $12 \%$ of the dogs with vagal stimulation $(p<0.001)$. In addition, we demonstrated that in half of the dogs studied, this antifibrillatory effect was independent of the heart rate reduction. This finding was essential for the subsequent human studies, such as the one on heart failure to be discussed now.

Subsequently, two large studies $[11,12]$ in patients with heart failure demonstrated first an inverse relationship between NYHA class and baroreflex sensitivity and, more importantly, a significantly higher mortality for patients with impaired vagal reflexes and, later, that this predictive value was present also among patients treated with beta-blockers. We realised that vagal stimulation had the potential of being of value, also for heart failure patients. Meanwhile, a series of experimental studies [13-16], beginning with the one by Kenji Sunagawa's group in Japan [13], were published and they all indicated that vagal stimulation was of major benefit in animal models of heart failure.

These clinical and experimental studies, taken together, provided a strong rationale for the first study with vagal stimulation in humans [17]. We started with a single-centre feasibility and safety study [17] and then moved to a pilot multicentre study [18]. Vagal stimulation was performed using an implantable neurostimulator system (CardioFit 500; BioControl Medical Ltd.) capable of delivering low current electrical pulses, with adjustable parameters to stimulate the vagus nerve. The stimulator was designed to sense heart rate (via an intracardiac electrode) and deliver stimulation at a fixed delay $(70 \mathrm{~ms})$ from the $\mathrm{R}$ wave. The stimulator microprocessor responds to the sensed heart rate and can adjust stimulation accordingly.

The single-centre phase II feasibility study in patients with severe congestive heart failure represented the first in-man study of chronic vagal stimulation for the management of a cardiac condition. The initial data on 8 patients suggested that this novel approach was feasible and appeared safe. We then moved to a larger and international multicentre study and enrolled 32 patients [18] with a history of congestive heart failure in NYHA class II-III, with a left ventricular ejection fraction (LVEF) $\leq 35 \%$, and with a heart rate on a 24-h Holter recording between 60 and 110 beats/min, on optimal medical treatment without changes in the previous 3 months. At the time of implant of the neurostimulator, the patients responded to vagal stimulation with a mean heart rate decrease of 7 beats $/$ min. During the 6-month duration of the study there were two serious adverse events related to the procedure but without consequences, whereas the other side effects were reduced or disappeared after fine-tuning of the stimulation intensity. There were also two deaths due to worsening of heart failure.

There were statistically significant improvements, at 6 months, in all main parameters under examination such as the NYHA classes which improved with 10 patients shifting to Class I, the 6-min walk test, and the Quality of Life test (Minnesota). Heart rate decreased from $82 \pm 13$ to $76 \pm 13$ beats $/ \min (p=0.03)$, the left ventricular end-systolic volume index from $103 \pm 35$ to $89 \pm 38 \mathrm{~mL} / \mathrm{m}^{2}(p=0.02)$, and LVEF from $22 \pm 7 \%$ to $29 \pm 8 \%(p=0.01)$. At 1 -year followup we had data on 23 patients. These data, based on internal control analysis, importantly show that all the changes observed at 6 months were preserved and remained statistically significant at 1-year follow-up. Of special note, LVEF for these 23 patients increased from $21 \pm 7 \%$ at baseline to $34 \pm$ $12 \%$ at 1 -year $(p<0.0001)$.

From these data we concluded that chronic vagal stimulation is safe and tolerable in symptomatic patients with congestive heart failure with marked clinical subjective improvements associated with objective long-lasting favourable changes. The preservation of these effects at 1-year follow-up strongly argues against a major role of a possible placebo effect.

Several mechanisms of action may have contributed to the beneficial effects of vagal stimulation in patients with heart failure. The modest changes in heart rate are unlikely to have played an important role in mediating the clinical effects and two studies in heart failure models found beneficial effects of vagal stimulation in the absence of any heart rate change [14, 15]. Heart rate-independent effects may include anti-adrenergic effects at ventricular level due to sympathetic-parasympathetic interaction, anti-apoptotic effects and the anti-inflammatory reflex postulated by Tracey [19]. In a model of ischaemia 
reperfusion in rats, vagal stimulation markedly reduced infarct size and markers of inflammation without changes in heart rate [20].

From this small multicentre study we concluded that it was time to design a controlled clinical trial with an adequate patient population aimed at definitely assessing whether or not chronic vagal stimulation may usefully represent a novel non-pharmacological approach to the treatment of symptomatic patients with heart failure. This is now being done with the ongoing trial called INOVATE-HF [21], which aims at enrolling up to 650 patients from 80 sites and to randomise them in a $3: 2$ ratio in order to receive either active treatment or standard optimal medical therapy.

Overall, this sequence of experimental and clinical studies, not unmindful of the role of the autonomic nervous system in heart failure [1], seems to represent a good example of translational cardiology.

\section{References}

1. Schwartz PJ, De Ferrari GM. Sympathetic-parasympathetic interaction in health and disease: abnormalities and relevance in heart failure. Heart Fail Rev. 2011;16:101-7.

2. De Ferrari GM, Schwartz PJ. Vagus nerve stimulation: from preclinical to clinical application: challenges and future directions. Heart Fail Rev. 2011;16:195-203.

3. Schwartz PJ. Vagal stimulation for the treatment of heart failure: a translational success story. Heart. 2012;98:1687-9.

4. Malliani A, Schwartz PJ, Zanchetti A. A sympathetic reflex elicited by experimental coronary occlusion. Am J Physiol. 1969;217:703-9.

5. Schwartz PJ, Pagani M, Lombardi F, et al. A cardiocardiac sympatho-vagal reflex in the cat. Circ Res. 1973;32:215-20.

6. Billman GE, Schwartz PJ, Stone HL. The effects of daily exercise on susceptibility to sudden cardiac death. Circulation. 1984;69:1182-9.

7. La Rovere MT, Bigger Jr JT, Marcus FI, et al. For the ATRAMI Investigators: Baroreflex sensitivity and heart-rate variability in prediction of total cardiac mortality after myocardial infarction. Lancet. 1998;351:478-84.
8. Levy MN, Schwartz PJ, editors. Vagal control of the heart: experimental basis and clinical implications. Armonk: Futura Publishing Co; 1994. p. 644.

9. Myers RW, Pearlman AS, Hyman RM, et al. Beneficial effect of vagal stimulation and bradycardia during experimental acute myocardial ischemia. Circulation. 1974;49:943-7.

10. Vanoli E, De Ferrari GM, Stramba-Badiale M, et al. Vagal stimulation and prevention of sudden death in conscious dogs with a healed myocardial infarction. Circ Res. 1991;68:1471-81.

11. Mortara A, La Rovere MT, Pinna GD, et al. Arterial baroreflex modulation of heart rate in chronic heart failure: clinical and hemodynamic correlates and prognostic implications. Circulation. 1997;96:3450-8.

12. La Rovere MT, Pinna GD, Maestri R, et al. Prognostic implications of baroreflex sensitivity in heart failure patients in the betablocking era. J Am Coll Cardiol. 2009;53:193-9.

13. Li M, Zheng C, Sato T, et al. Vagal nerve stimulation markedly improves long-term survival after chronic heart failure in rats. Circulation. 2004;109:120-4.

14. Sabbah HN, Rastogi S, Mishra S, et al. Long-term therapy with neuroselective electric vagus nerve stimulation improves LV function and attenuates global LV remodelling in dogs with chronic heart failure. (abstr). Eur J Heart Fail. 2005;4(Suppl):166.

15. Sabbah HN, Imai M, Zaretsky A, et al. Therapy with vagus nerve electrical stimulation combined with beta-blockade improves left ventricular systolic function in dogs with heart failure beyond that seen with beta-blockade alone. (abstr). Eur J Heart Fail. 2007;6 Suppl 1:114.

16. Zhang Y, Popovic ZB, Bibevski S, et al. Chronic vagus nerve stimulation improves autonomic control and attenuates systemic inflammation and heart failure progression in a canine high-rate pacing model. Circ Heart Fail. 2009;2:692-9.

17. Schwartz PJ, De Ferrari GM, Sanzo A, et al. Long term vagal stimulation in patients with advanced heart failure: first experience in man. Eur J Heart Fail. 2008;10:884-91.

18. De Ferrari GM, Crijns HJGM, Borggrefe M, et al. Chronic vagus nerve stimulation: a new and promising therapeutic approach for chronic heart failure. Eur Heart J. 2011;32:847-55.

19. Tracey KJ. The inflammatory reflex. Nature. 2002;420:853-9.

20. Calvillo L, Vanoli E, Andreoli E, et al. Vagal stimulation, through its nicotinic action, limits infarct size and the inflammatory response to myocardial ischemia and reperfusion. J Cardiovasc Pharmacol. 2011;58:500-7.

21. Hauptman PJ, Schwartz PJ, Gold MR, et al. Rationale and study design of the increase of vagal tone in heart failure study: INOVATE-HF. Am Heart J. 2012;163:954-62. 